\title{
Optimal control of the dengue dynamical transmission with vertical transmission
}

\author{
Puntani Pongsumpun ${ }^{1 *}$ (D) I.-Ming Tang ${ }^{2}$ and Napasool Wongvanich ${ }^{3}$ (D)
}

${ }^{\text {"Correspondence: }}$

kppuntan@kmitl.ac.th

'Department of Mathematics, Faculty of Science, King Mongkut's Institute of Technology Ladkrabang, Bangkok, Thailand

Full list of author information is available at the end of the article

\section{Springer}

\begin{abstract}
Dengue disease is found in tropical and subtropical regions around the world. Dengue virus is the cause of dengue fever, dengue hemorrhagic fever, and dengue shock syndrome. It consists of 4 serotypes: DEN-1, DEN-2, DEN-3, and DEN-4. There are two modes of transmission for dengue virus in mosquito: horizontal transmission and vertical transmission. The mosquito can be infected when it bites an infectious human by horizontal transmission, but there can also be vertical transmission through sexual contact with an infected mosquito. This research presents a control mechanism based on our previously developed dengue model with vertical transmission. The two policies, namely vaccination and insecticide administration (Policy 1) and isolation and insecticide administration (Policy 2) are considered. The use of Pontryargin's maximum principle allowed necessary and optimality conditions, thus facilitating the optimal control to be developed. Numerical solutions of our control systems and the conclusions of our two policies are presented.
\end{abstract}

Keywords: Dengue fever; Optimal control; Vertical transmission; Epidemiological control

\section{Introduction}

The Dengue disease is the mosquito-borne viral infection. There are three forms of dengue infection [1]: dengue fever (DF), dengue hemorrhagic fever (DHF), and dengue shock syndrome (DSS). The symptoms of simple DF are a high fever, aching muscles and joints, pain behind the eyes, and a body rash that can disappear and then reappear. The above symptoms normally disappear after one week. Some dengue fever patients may develop more severe forms of the disease. DHF can be accompanied by severe bleeding, where these patients can go into severe shock and die. This phase of the disease is labeled DSS [2]. There are four serotypes (DEN-1, DEN-2, DEN-3, and DEN-4) of the dengue virus. Infection with any one serotype will produce long term immunity to that serotype but short term immunity to the other three serotypes [3]. More than 100 countries in the regions of the Americas, Africa, the Eastern Mediterranean, South-East Asia, and the Western Pacific are the endemic regions of this disease.

Dengue virus is transmitted among humans through the bite of infectious Aedes mosquitoes. An infected mosquito carries the DF virus but cannot pass the virus until it becomes infectious. The cycle of dengue transmission begins with a dengue infectious person. Each infectious person has virus circulating in the blood or viremia. This stage usually lasts for about 5-12 days. When a susceptible female Aedes mosquito bites an

(c) The Author(s) 2019. This article is distributed under the terms of the Creative Commons Attribution 4.0 International License (http://creativecommons.org/licenses/by/4.0/), which permits unrestricted use, distribution, and reproduction in any medium, provided you give appropriate credit to the original author(s) and the source, provide a link to the Creative Commons license, and indicate if changes were made. 
infectious person, the virus in the blood will be passed to the mosquito. Within the susceptible mosquito, the viruses will replicate during an extrinsic incubation period of 8-12 days, depending on the temperature. After this period, the female mosquito becomes infectious, in other words, able to transmit the virus to a human. This is called horizontal transmission [4].

Since the blood is needed for the development of the egg, biting of humans is done by the female mosquitoes [5]. The male mosquitoes feed on the nectar of plants. Since the advent of a very sensitive detection of the DNA of the dengue virus, the DNA testing has been done on a collection of male mosquitoes [6,7]. Because the male mosquitoes do not feed on the blood of infectious human beings, it was suggested that the dengue virus DNA detected in the male was from the virus obtained by sexual contact with the infectious female mosquito. This opens up the possibility that a susceptible female can become infected by the same type of contact with the infectious male mosquitoes. This type of transmission is known as transovarial vertical transmission (VT). To quantify the VT rates, Clements et al. measured the effective rates of VT in Aedes and Culex mosquitoes [8]. Their results demonstrated that, the Aedes eggs displayed higher effective VT rates and are generally more resistant to desiccation than the Culex eggs, which may confer a selective advantage to vertically transmitted viruses [8]. While the recent work of Sanchez-Vargas [7] suggests that VT is a potential mechanism of maintaining the dengue virus during inter-epidemic periods.

With much fanfare, Sanofi Pasteur announced in 2016 the development of a live recombinant tetravalent dengue vaccine Dengvaxia. Dengvaxia underwent phase III clinical trials and passed them [9]. Based on the findings, several countries, among them were Brazil and the Philippines, began an extensive vaccination program. Initial results were encouraging [10]. However, with the passage of time, trouble began to appear so that on December 12, 2007, the Philippines Department of Health suspended the program [11] as did most other countries. WHO [12] issued new guidelines about the usage of Dengvaxia as part of any vaccination program for dengue fever. The trouble with the vaccine concerns antibody-dependent enhancement (ADE) of antigens of the non-prevalent strains in the community of the vaccine.

Before the non-success of the Dengvaxia vaccine, two classical policies have been proposed to reduce the incidence of infections by the dengue virus: Policy 1 involves vaccination and insecticide administration, while Policy 2 involves isolation or quarantine and insecticide administration. Policy 2 is the least expensive option, but in some countries involves the violation of the human right of being able to move around even if one does not exhibit any symptoms of the illness. An example of this is furore in the Sierra Leone, where "escapees" from the quarantine program have been publicly named and shamed by the national authorities [13]. Another example of the furore is in the USA when a white women nurse returned to the USA after working with patients who had been infected with Ebola. The nurse who had not exhibited any symptoms was put into a tent at the airport and kept in isolation for several weeks. In the USA, human rights override any steps taken for the "greater" good, the protection of society as a whole.

Since no clinical studies can be executed to see whether quarantine of people exposed to Ebola or the dengue viruses help in stopping these epidemics, answering this question thus relies on the computer simulations facilitated by mathematical modeling and control theories, which have been carried out by researchers around the world. A number of 
mathematical models describing the dynamics of the dengue virus transmission have been proposed in the literature. These models include assumptions of constant total human and vector populations [14, 15]; variable total human population [16]; dengue virus reinfection of the same serotype [17]; dengue virus transmission with memory [18]; possibility of vertical transmission of the dengue virus [19]. Yang and Lee [20] studied the effects of controlling vectors on the transmission of the disease between the mosquitoes and the humans. The works of Al-Sulami and Hamdan et al. [21,33] recently introduced a fractional order dengue epidemic model, while Iboi and Gumel [22] evaluated mathematically the role of the Dengvaxia vaccine. Similar mathematical models have also been proposed in the literature to describe the dynamics of the Ebola and Rubella fevers [23-25]. Rodrigues et al. in 2010 [26] proposed that optimal control theory be used to determine the parameters involved in the control of the mosquito which lead to optimal decrease in the number of infectious mosquitoes over a short time and at less cost. Recently, Imran [27] proposed the strategy of optimal control to reduce the Zika cases. They found that the optimal control strategy is the most useful to eliminate the disease and the cost. Momoh and Fuegenschuh [28] applied the optimal control theory on the Zika virus model and compared the economic effectiveness of common measures such as bednets, condoms, and indoor residual spray. Although these works have considered the use of optimal control to reduce virulent infectious diseases, the underlying mathematical models used were just the basic SEIR model where the effects of vertical transmission have not been taken into account; whereas others concentrated only a single policy [27-29]. In this paper, we apply the optimal control on the model of Chanprasopchai et al. [19] which includes vertical transmission, that is becoming increasingly important due to the works of Clements and Sanchez-Vargas [7, 8], and analyzes the outcomes of the control implementation on both policies.

\section{Methodology}

\subsection{The mathematical model}

The mathematical model governing the dynamics of the dengue disease is developed, incorporating the effects of vector-host dynamics. In this respect the model itself is developed for both the human and the mosquito vector. Here an SEIR model is considered for the human population, where the total human population is divided into four classes, namely $\tilde{S}_{H}, \tilde{E}_{H}, \tilde{I}_{H}$, and $\tilde{R}_{H}$. The mosquito vector population is subdivided into three compartments, namely $\tilde{S}_{V}, \tilde{E}_{V}, \tilde{I}_{V}$. The associated transmission diagram between the relevant compartments is taken from the previous work of Chanprasopchai et al. [19]. The transmission diagram duly admits the following system of differential equations defined:

$$
\begin{aligned}
& \tilde{S}_{H}^{\prime}=\lambda_{H} N_{H}-\frac{b \beta_{H}}{N_{H}} \tilde{S}_{H} \tilde{I}_{V}-\mu_{H} \tilde{S}_{H}, \\
& \tilde{E}_{H}^{\prime}=\frac{b \beta_{H}}{N_{H}} \tilde{S}_{H} \tilde{I}_{V}-\left(\epsilon_{H}+\mu_{H}\right) \tilde{E}_{H}, \\
& \tilde{I}_{H}^{\prime}=\epsilon_{H} \tilde{E}_{H}-\left(r_{H}+\mu_{H}\right) \tilde{I}_{H}, \\
& \tilde{R}_{H}^{\prime}=r_{H} \tilde{I}_{H}-\mu_{H} \tilde{R}_{H}, \\
& \tilde{S}_{V}^{\prime}=A-\frac{b \beta_{V}}{N_{H}} \tilde{S}_{V} \tilde{I}_{H}-\mu_{V} \tilde{S}_{V}, \\
& \tilde{E}_{V}^{\prime}=\frac{b \beta_{V}}{N_{H}} \tilde{S}_{V}-\left(\epsilon_{H}+\mu_{H}\right) \tilde{E}_{H},
\end{aligned}
$$




$$
\tilde{I}_{V}^{\prime}=M+\epsilon_{V} \tilde{E}_{V}-\mu_{V} \tilde{I}_{V}
$$

where the assumptions are

$$
\begin{aligned}
& N_{H}=\tilde{S}_{H}+\tilde{E}_{H}+\tilde{I}_{H}+\tilde{R}_{H}, \\
& N_{V}=\tilde{S}_{V}+\tilde{E}_{V}+\tilde{I}_{V} .
\end{aligned}
$$

The variables of Equations (1)-(9) are defined as follows:

$\tilde{S}_{H}$ denotes the number of susceptible human individuals at time $t$;

$\tilde{E}_{H}$ denotes the number of exposed human individuals at time $t$;

$\tilde{I}_{H}$ denotes the number of infected human individuals at time $t$;

$\tilde{R}_{H}$ denotes the number of recovered human individuals at time $t$;

$\tilde{S}_{V}$ denotes the number of susceptible vector population at time $t$;

$\tilde{E}_{V}$ denotes the number of exposed vector population at time $t$;

$\tilde{I}_{V}$ denotes the number of infected vector population at time $t$;

$A$ denotes the constant recruitment rate;

$M$ denotes the number of mosquitoes transovarially infected;

$N_{H}$ denotes the total number of human population;

$N_{V}$ denotes the total number of vector population.

The parameters of Equations (1)-(7) are defined as given in Table 1. Note that the infection rate does not introduce exogenous deaths in the population, and also that since the infection time is assumed to be minimal, the population is thus assumed to be constant for all time $t$. Consequently, the rate of change of both the total population of human and vectors is zero, symbolically:

$$
\begin{aligned}
& \frac{d \tilde{S}_{H}}{d t}+\frac{d \tilde{E}_{H}}{d t}+\frac{d \tilde{I}_{H}}{d t}+\frac{d \tilde{R}_{H}}{d t}=0 \\
& \frac{d \tilde{S}_{V}}{d t}+\frac{d \tilde{E}_{V}}{d t}+\frac{d \tilde{I}_{V}}{d t}=0 .
\end{aligned}
$$

\begin{tabular}{|c|c|}
\hline Parameter & Definition \\
\hline$\overline{\lambda_{H}}$ & Per capita birth rate of the human population \\
\hline$b$ & Biting rate of the human population \\
\hline$\beta_{H}$ & $\begin{array}{l}\text { Transmission probability of dengue virus from the } \\
\text { vector population to the human population }\end{array}$ \\
\hline$\beta_{V}$ & $\begin{array}{l}\text { Transmission probability of dengue virus from the } \\
\text { human population to the vector population }\end{array}$ \\
\hline$\epsilon_{H}$ & Intrinsic incubation rate \\
\hline$\epsilon_{V}$ & Extrinsic incubation rate \\
\hline$\mu_{H}$ & Death rate of the human population \\
\hline$\mu_{V}$ & Death rate of the vector population \\
\hline$r_{H}$ & Recovery rate of the vector population \\
\hline
\end{tabular}

Table 1 Parameter definition of the differential system of Equations (1)-(7) 
From the last boundary conditions, we have

$$
N_{V}=\frac{A+M}{\mu_{V}}
$$

and

$$
\mu_{H}=\beta_{H} .
$$

Defining normalized compartmental variables as follows:

$$
\begin{array}{lll}
S_{H}=\frac{\tilde{S}_{H}}{N_{H}}, & E_{H}=\frac{\tilde{E}_{H}}{N_{H}}, & I_{H}=\frac{\tilde{I}_{H}}{N_{H}}, \\
S_{V}=\frac{\tilde{S}_{V}}{N_{V}}, & E_{V}=\frac{\tilde{E}_{V}}{N_{V}}, & I_{V}=\frac{\tilde{R}_{H}}{N_{H}}, \\
N_{V} &
\end{array}
$$

we have

$$
\begin{aligned}
& S_{H}+E_{H}+I_{H}+R_{H}=1, \\
& S_{V}+E_{V}+I_{V}=1 .
\end{aligned}
$$

In terms of the normalized compartments, the differential equations become:

$$
\begin{aligned}
& S_{H}^{\prime}=\mu_{H}\left(1-S_{H}\right)-\frac{b \beta_{H}}{N_{H}} S_{H} I_{V} N_{V}, \\
& E_{H}^{\prime}=\frac{b \beta_{H}}{N_{H}} S_{H} I_{V} N_{V}-\left(\epsilon_{H}+\mu_{H}\right) E_{H}, \\
& I_{H}^{\prime}=\epsilon_{H} E_{H}-\left(\mu_{H}+r_{H}\right) I_{H}, \\
& E_{V}^{\prime}=b \beta_{V} S_{V} I_{H}-\left(\epsilon_{V}+\mu_{V}\right) E_{V}, \\
& I_{V}^{\prime}=\frac{M}{N_{V}}+\epsilon_{V} E_{V}-\mu_{V} I_{V} .
\end{aligned}
$$

\subsection{Stability analysis}

\subsubsection{System under the presence of vertical transmission}

When vertical transmission is possible, the system is governed by Equations (17)-(21). Setting the right-hand sides of these equations to zeros, the equilibrium states can be obtained. We find that the only equilibrium state that is possible is the endemic equilibrium state $E_{1}$ given by

$$
E_{1}=\left\{S_{H}^{1 *}, E_{H}^{1 *}, I_{H}^{1 *}, E_{V}^{1 *}, I_{V}^{1 *}\right\}
$$

where the equilibrium states $S_{H}^{1 *}, E_{H}^{1 *}, I_{H}^{1 *}, E_{V}^{1 *}, I_{V}^{1 *}$ are given by Equations (24)-(28) in the work of Chanprasopchai et al. [19].

Note that the value of the basic reproduction number $R_{0}$ is given by

$$
\begin{aligned}
R_{0}= & \left(\alpha_{1}+N_{V} M \alpha_{2}\left(\gamma_{H} \alpha_{3}+\mu_{H}\left(\alpha_{4}+\alpha_{3}\right)\right)\right) \\
& +\left[N _ { V } ^ { 2 } \left(\alpha_{5} \alpha_{2} \alpha_{6} \alpha_{3}^{2}\left(\alpha_{4} \mu_{H}+\alpha_{5} \alpha_{7} \mu_{V}\right)\right.\right.
\end{aligned}
$$




$$
\left.+\frac{\left.\left.\left.\left(\alpha_{8} \alpha_{6} \alpha_{3}-\alpha_{2}\left(M \gamma_{H} \alpha_{3}+\mu_{H}\left(\alpha_{4} \alpha_{9}+M \alpha_{3}\right)\right)\right)^{2}\right)\right)\right)}{N_{V} \alpha_{8} \alpha_{6} \alpha_{3}}\right]^{1 / 2},
$$

where

$$
\begin{aligned}
& \alpha_{1}=b^{2} N_{V}^{2} \beta_{H} \beta_{V} \epsilon_{H} \epsilon_{V} \mu_{H}, \quad \alpha_{2}=b \beta_{H}, \quad \alpha_{3}=\left(\epsilon_{H}+\mu_{H}\right)\left(\epsilon_{V}+\mu_{V}\right), \\
& \alpha_{4}=b \beta_{V} \epsilon_{H}, \quad \alpha_{5}=4 n_{H} \mu_{H} M, \quad \alpha_{6}=\gamma_{H}+\mu_{H}, \quad \alpha_{7}=\epsilon_{H}+\mu_{H}, \\
& \alpha_{8}=N_{H} \mu_{H} \mu_{V}, \quad \alpha_{9}=M+N_{V} \epsilon_{V} .
\end{aligned}
$$

Proposition 1 The equilibrium state $E_{1}$ of Equation (22) is asymptotically stable when $R_{0}$ is above unity.

Proof See the proof of Proposition 1 in [19].

\subsubsection{System under the absence of vertical transmission}

For the system under the absence of vertical transmission, the number of mosquitoes transovarially infected $M$ is set to zero. This system now admits two equilibrium points, namely a disease free equilibrium point and an endemic equilibrium point. Specifically, the equilibrium points will occur at:

1. Disease free equilibrium

$$
E_{0}=(1,0,0,0,0)^{T},
$$

2. Endemic equilibrium

$$
E_{2}=\left\{S_{H}^{2 *}, E_{H}^{2 *}, I_{H}^{2 *}, E_{V}^{2 *}, I_{V}^{2 *}\right\}
$$

where $S_{H}^{2 *}, E_{H}^{2 *}, I_{H}^{2 *}, E_{V}^{2 *}, I_{V}^{2 *}$ are given in Equations (38)-(42) of [19].

Proposition 2 The equilibrium state $E_{0}$ of Equation (22) is asymptotically stable when $R_{0}$ is below unity.

Proof See the proof of Proposition 2 in [19].

Proposition 3 The equilibrium state $E_{2}$ of Equation (22) is asymptotically stable when $R_{0}>1$.

Proof See the proof of Proposition 3 in [19].

\section{General setting of the optimal control problem}

Equations (17)-(21) can be recasted as a control problem, the aim of which is to minimize the number of infected human population. Since the system includes the dynamics for both the human and the vector populations, two control inputs can be attributed, namely $u_{1}$ for the human population and $u_{2}$ for the mosquito population. Under the action of $u_{1}$, two possible policies are considered: vaccination and isolation. The action of $u_{2}$ is the insecticide control effort. Note that both control inputs are assumed to be piecewise continuous functions taking values in a positive bounded set $U=\left[0, u_{\max }\right]$. We apply the 
different control policies separately by adding a linear term in the control variable $u_{i}(t)$ to the reduced system of Equations (17)-(21).

Policy 1 Vaccination and insecticide administration.

It is expected that under the action of $u_{1}$, the human susceptibles will be removed from the system. However, vaccination only plays a weak role on the exposed and infected populations, and it is thus assumed that $u_{1}$ will have no effect on $E_{H}$ and $I_{H}$. The insecticide administration will remove the mosquito population; and consequently, it is assumed that $u_{2}$ will act on both $E_{V}$ and $I_{V}$. The control model under Policy 1 is expressed by the set of equations:

$$
\begin{aligned}
& S_{H}^{\prime}=\mu_{H}\left(1-S_{H}\right)-\frac{b \beta_{H}}{N_{H}} S_{H} I_{V} N_{V}-u_{1}(t) S_{H}, \\
& E_{H}^{\prime}=\frac{b \beta_{H}}{N_{H}} S_{H} I_{V} N_{V}-\left(\epsilon_{H}+\mu_{H}\right) E_{H}, \\
& I_{H}^{\prime}=\epsilon_{H} E_{H}-\left(\mu_{H}+r_{H}\right) I_{H}, \\
& E_{V}^{\prime}=b \beta_{V} S_{V} I_{H}-\left(\epsilon_{V}+\mu_{V}+u_{2}(t)\right) E_{V}, \\
& I_{V}^{\prime}=\frac{M}{N_{V}}+\left(\epsilon_{V}-u_{2}(t)\right) E_{V}-\left(\mu_{V}+u_{2}(t)\right) I_{V} .
\end{aligned}
$$

Policy 2 Isolation and insecticide administration.

Here, the action of the isolation control $u_{1}$ is to anticipate the removal of the infected human individuals from the system. However, isolation only plays a minor role on the susceptible and exposed populations, and it is thus assumed that $u_{1}$ will have no effect on $S_{H}$ and $E_{H}$. The insecticide administration achieves similar effects to those of Policy 1 , and consequently it is again assumed that $u_{2}$ will act on both $E_{V}$ and $I_{V}$. The control model under Policy 2 is as follows:

$$
\begin{aligned}
& S_{H}^{\prime}=\mu_{H}\left(1-S_{H}\right)-\frac{b \beta_{H}}{N_{H}} S_{H} I_{V} N_{V}, \\
& E_{H}^{\prime}=\frac{b \beta_{H}}{N_{H}} S_{H} I_{V} N_{V}-\left(\epsilon_{H}+\mu_{H}\right) E_{H}, \\
& I_{H}^{\prime}=\epsilon_{H} E_{H}-\left(\mu_{H}+r_{H}+u_{1}(t)\right) I_{H}, \\
& E_{V}^{\prime}=b \beta_{V} S_{V} I_{H}-\left(\epsilon_{V}+\mu_{V}+u_{2}(t)\right) E_{V}, \\
& I_{V}^{\prime}=\frac{M}{N_{V}}+\left(\epsilon_{V}-u_{2}(t)\right) E_{V}-\left(\mu_{V}+u_{2}(t)\right) I_{V} .
\end{aligned}
$$

The optimal control problems of Equations (29)-(33) and (34)-(38), require a definition of the objective function as follows:

$$
J\left(u_{1}, u_{2}\right)=\int_{0}^{T}\left(B_{0} I_{H}(t)+\frac{1}{2} B_{1} u_{1}^{2}+\frac{1}{2} B_{2} u_{2}^{2}\right) d t
$$

subjected to the systems of Equations (29) -(33) for the first policy and Equations (34) - (38) for the second policy. The weight $B_{0}$ is associated with the human infective population. 
Note that we are only interested in minimizing the infected human individuals $I_{H}$ and not the mosquito compartments. The weights $B_{1}$ and $B_{2}$ are associated with the control variables $u_{1}$ and $u_{2}$ respectively.

\subsection{The existence of optimal control}

The existence of the optimal control for both policies can be proven using the results given in the works of Fleming and Rishel and the references therein [30]. According to the results in these works, the Lagrangian for the optimal control problems of Equations (29)-(33) and (34) $-(38)$ is

$$
L\left(I_{H}, u_{1}, u_{2}\right)=B_{0} I_{H}(t)+\frac{1}{2} B_{1} u_{1}^{2}+\frac{1}{2} B_{2} u_{2}^{2} .
$$

Theorem 4 There exists an optimal control pair $u_{1}^{*}(t)$ and $u_{2}^{*}(t)$ so that

$$
J\left(u_{1}^{*}, u_{2}^{*}\right)=\min _{u_{1}, u_{2} \in U} J\left(u_{1}, u_{2}\right) .
$$

Proof In order to prove Theorem 4, it suffices to check the following properties:

1. The corresponding set of controls and the state variables is nonempty.

2. The control set $U$ is convex and closed.

3. The right-hand side of the state system is bounded by the linear function in the state and control variables.

4. The integrand of the objective function is convex on $U$.

5. There exist nonnegative constants $c_{1}$ and $c_{2}$ and $\rho>1$ satisfying the following expression:

$$
L\left(\mathbf{x}, u_{1}, u_{2}\right) \geq c_{2}+c_{1}\left(u_{1}^{\rho}+u_{2}^{\rho}\right) .
$$

We proceed to checking the following conditions:

1. The existence of the systems in Equations (29)-(33) and (34)-(38) is given with bounded coefficients, which satisfies Condition 1, according to Theorem 9.2.1 from Lukes [31].

2. From Condition 1, the control set is convex and closed, hence giving Condition 2.

3. Note that the state system is linear in $u_{1}$ and $u_{2}$, therefore the right-hand side of Equations (29)-(33) and (34)-(38) will satisfy Condition 3.

4. Since the solution to the systems of Equations (29)-(33) and (34)-(38) is bounded, the control functional is convex in $U$, giving Condition 4.

5. To prove Condition 5, let $\bar{c}_{2}=\min \left(I_{H}(t)\right)$ and $c_{1}=\min \left(B_{1}, B_{2}\right)$ and $\rho=2$, then the Lagrangian $L$ can be rewritten as

$$
\begin{aligned}
L\left(\mathbf{x}, u_{1}, u_{2}\right) & =B_{0} I_{H}(t)+\frac{1}{2} B_{1} u_{1}^{2}+\frac{1}{2} B_{2} u_{2}^{2} \\
& \geq B_{0} \bar{c}_{2}+c_{1}\left(\left|u_{1}\right|^{2}+\left|u_{2}\right|^{2}\right) \\
& =c_{2}+c_{1}\left(\left|u_{1}\right|^{2}+\left|u_{2}\right|^{2}\right) .
\end{aligned}
$$

All conditions are thus satisfied; as a consequence, there exists an optimal control for the systems of Equations (29)-(33) and (34)-(38). 


\subsection{Characterization of the optimal control}

The optimal control for both policies can be derived through the use of Pontryagin's maximum principle [32].

Theorem 5 There exist the adjoint variables $\lambda_{i}, i=1, \ldots, 5$, under the control of Policy 1 that satisfy the following:

$$
\begin{aligned}
& \frac{d \lambda_{1}}{d t}=-\lambda_{1}(t)\left(-\mu_{H}-\frac{b \beta_{H} N_{V}}{N_{H}} I_{V}-u_{1}^{*}\right)-\frac{b \beta_{H} N_{V}}{N_{H}} I_{V} \lambda_{2}(t), \\
& \frac{d \lambda_{2}}{d t}=-\lambda_{2}(t)\left(-\epsilon_{H}-\mu_{H}\right)-\lambda_{3}(t) \epsilon_{H}, \\
& \frac{d \lambda_{3}}{d t}=-\lambda_{3}(t)\left(-\mu_{H}-r_{H}\right)-b \beta_{V} S_{V} \lambda_{4}(t)-B_{0}, \\
& \frac{d \lambda_{4}}{d t}=-\lambda_{4}(t)\left(-\epsilon_{V}-\mu_{V}-u_{2}^{*}\right)-\lambda_{5}(t)\left(\epsilon_{V}-u_{2}^{*}\right), \\
& \frac{d \lambda_{5}}{d t}=\frac{b \beta_{H} S_{H} N_{V}}{N_{H}}\left(\lambda_{1}(t)-\lambda_{2}(t)\right)-\lambda_{5}(t)\left(-\mu_{V}-u_{2}^{*}\right)
\end{aligned}
$$

with the boundary conditions

$$
\lambda_{i}(T)=0 \quad \text { for all } i=1, \ldots, 5 .
$$

In addition, the optimal control variables are given by

$$
\begin{aligned}
& u_{1}^{*}(t)=\max \left(\min \left(\frac{\lambda_{1} S_{H}^{*}}{B_{1}}, u_{1}^{\max }\right), 0\right), \\
& u_{2}^{*}(t)=\max \left(\min \left(\frac{E_{V}^{*} \lambda_{4}+\lambda_{5}\left(I_{V}^{*}+E_{V}\right)}{B_{2}}, u_{2}^{\max }\right), 0\right) .
\end{aligned}
$$

Proof The Hamiltonian for the optimal control of Policy 1 is defined as follows:

$$
\begin{aligned}
H= & L\left(\mathbf{x}, u_{1}, u_{2}\right)+\lambda_{1} \frac{d S_{H}}{d t}+\lambda_{2} \frac{d E_{H}}{d t}+\lambda_{3} \frac{d I_{H}}{d t}+\lambda_{4} \frac{d E_{V}}{d t}+\lambda_{5} \frac{d I_{V}}{d t} \\
= & B_{0} I_{H}(t)+\frac{1}{2} B_{1} u_{1}^{2}+\frac{1}{2} B_{2} u_{2}^{2}+\lambda_{1}\left\{\mu_{H}\left(1-S_{H}\right)\right. \\
& \left.-\frac{b \beta_{H}}{N_{H}} S_{H} I_{V} N_{V}-u_{1}(t) S_{H}\right\}+\lambda_{2}\left\{\frac{b \beta_{H}}{N_{H}} S_{H} I_{V} N_{V}-\left(\epsilon_{H}+\mu_{H}\right) E_{H}\right\} \\
& +\lambda_{3}\left\{\epsilon_{H} E_{H}-\left(\mu_{H}+r_{H}\right) I_{H}\right\}+\lambda_{4}\left\{b \beta_{V} S_{V} I_{H}-\left(\epsilon_{V}+\mu_{V}+u_{2}(t)\right) E_{V}\right\} \\
& +\lambda_{5}\left\{\frac{M}{N_{V}}+\left(\epsilon_{V}-u_{2}(t)\right) E_{V}-\left(\mu_{V}+u_{2}(t)\right) I_{V}\right\} .
\end{aligned}
$$

The adjoint system is obtained as follows:

$$
\begin{aligned}
& \frac{d \lambda_{1}}{d t}=-\frac{\partial H}{\partial S_{H}}=-\lambda_{1}(t)\left(-\mu_{H}-\frac{b \beta_{H} N_{V}}{N_{H}} I_{V}-u_{1}^{*}\right)-\frac{b \beta_{H} N_{V}}{N_{H}} I_{V} \lambda_{2}(t), \\
& \frac{d \lambda_{2}}{d t}=-\frac{\partial H}{\partial E_{H}}=-\lambda_{2}(t)\left(-\epsilon_{H}-\mu_{H}\right)-\lambda_{3}(t) \epsilon_{H},
\end{aligned}
$$




$$
\begin{aligned}
& \frac{d \lambda_{3}}{d t}=-\frac{\partial H}{\partial I_{H}}=-\lambda_{3}(t)\left(-\mu_{H}-r_{H}\right)-b \beta_{V} S_{V} \lambda_{4}(t)-B_{0}, \\
& \frac{d \lambda_{4}}{d t}=-\frac{\partial H}{\partial E_{V}}=-\lambda_{4}(t)\left(-\epsilon_{V}-\mu_{V}-u_{2}^{*}\right)-\lambda_{5}(t)\left(\epsilon_{V}-u_{2}^{*}\right), \\
& \frac{d \lambda_{5}}{d t}=-\frac{\partial H}{\partial I_{V}}=\frac{b \beta_{H} S_{H} N_{V}}{N_{H}}\left(\lambda_{1}(t)-\lambda_{2}(t)\right)-\lambda_{5}(t)\left(-\mu_{V}-u_{2}^{*}\right) .
\end{aligned}
$$

Using the optimality conditions, we find that

$$
\frac{\partial H}{\partial u_{j}}=0, \quad \text { for all } j=1,2 \text { at } u_{j}=u_{j}^{*}
$$

Hence,

$$
\begin{aligned}
& \frac{\partial H}{\partial u_{1}}=B_{1} u_{1}-\lambda_{1} S_{H}=0 \quad \Longrightarrow \quad u_{1}^{*}=\frac{\lambda_{1} S_{H}}{B_{1}}, \\
& \frac{\partial H}{\partial u_{2}}=B_{2} u_{2}-E_{V} \lambda_{4}-\lambda_{5}\left(-E_{V}-I_{V}\right)=0 \quad \Longrightarrow \quad u_{2}^{*}=\frac{E_{V} \lambda_{4}+I_{V} \lambda_{5}+E_{V} \lambda_{5}}{B_{2}} .
\end{aligned}
$$

Using the property of the control set, we can say that

$$
\begin{aligned}
& u_{1}^{*}= \begin{cases}0 & \text { if } \frac{\lambda_{1} S_{H}}{B_{1}} \leq 0, \\
\frac{\lambda_{1} S_{H}}{B_{1}} & \text { if } \frac{\lambda_{1} S_{H}}{B_{1}}<u_{1}^{\max }, \\
u_{1}^{\max } & \text { if } \frac{\lambda_{1} S_{H}}{B_{1}} \geq u_{1}^{\max },\end{cases} \\
& u_{2}^{*}= \begin{cases}0 & \text { if } \frac{E_{V} \lambda_{4}+I_{V} \lambda_{5}+E_{V} \lambda_{5}}{B_{2}} \leq 0, \\
\frac{E_{V} \lambda_{4}+I_{V} \lambda_{5}+E_{V} \lambda_{5}}{B_{2}} & \text { if } \frac{E_{V} \lambda_{4}+I_{V} \lambda_{5}+E_{V} \lambda_{5}}{B_{2}}<u_{2}^{\max }, \\
u_{2}^{\max } & \text { if } \frac{E_{V} \lambda_{4}+I_{V} \lambda_{5}+E_{V} \lambda_{5}}{B_{2}} \geq u_{2}^{\max } .\end{cases}
\end{aligned}
$$

Theorem 6 There exist adjoint variables $\lambda_{i}, i=1, \ldots, 5$, under the control of Policy 2 that satisfy the following:

$$
\begin{aligned}
& \frac{d \lambda_{1}}{d t}=-\lambda_{1}(t)\left(-\mu_{H}-\frac{b \beta_{H} N_{V} I_{V}}{N_{H}}\right)-\frac{b \beta_{H} N_{V}}{N_{H}} I_{V} \lambda_{2}(t), \\
& \frac{d \lambda_{2}}{d t}=-\lambda_{2}(t)\left(-\epsilon_{H}-\mu_{H}\right)-\lambda_{3}(t) \epsilon_{H}, \\
& \frac{d \lambda_{3}}{d t}=-\lambda_{3}(t)\left(-\mu_{H}-r_{H}-u_{1}^{*}\right)-b \beta_{V} S_{V} \lambda_{4}(t)-B_{0}, \\
& \frac{d \lambda_{4}}{d t}=-\lambda_{4}(t)\left(-\epsilon_{V}-\mu_{V}-u_{2}^{*}\right)-\lambda_{5}(t)\left(\epsilon_{V}-u_{2}^{*}\right), \\
& \frac{d \lambda_{5}}{d t}=\frac{b \beta_{H} S_{H} N_{V}}{N_{H}}\left(\lambda_{1}(t)-\lambda_{2}(t)\right)-\lambda_{5}(t)\left(-\mu_{V}-u_{2}^{*}\right)
\end{aligned}
$$

with the boundary conditions

$$
\lambda_{i}(T)=0 \quad \text { for all } i=1, \ldots, 5 \text {. }
$$


In addition, the optimal control variables are given by

$$
\begin{aligned}
& u_{1}^{*}(t)=\max \left(\min \left(\frac{\lambda_{1} S_{H}^{*}}{B_{1}}, u_{1}^{\max }\right), 0\right), \\
& u_{2}^{*}(t)=\max \left(\min \left(\frac{\lambda_{4} E_{V}+\lambda_{5}\left(I_{V}+E_{V}\right)}{B_{2}}, u_{2}^{\max }\right), 0\right) .
\end{aligned}
$$

Proof The proof proceeds in a similar fashion as was done for the optimal control under Policy 1. The Hamiltonian is defined as follows:

$$
\begin{aligned}
H= & B_{0} I_{H}(t)+\frac{1}{2} B_{1} u_{1}^{2}+\frac{1}{2} B_{2} u_{2}^{2}+\lambda_{1}\left\{\mu_{H}\left(1-S_{H}\right)-\frac{b \beta_{H}}{N_{H}} S_{H} I_{V} N_{V}\right\} \\
& +\lambda_{2}\left\{\frac{b \beta_{H}}{N_{H}} S_{H} I_{V} N_{V}-\left(\epsilon_{H}+\mu_{H}\right) E_{H}\right\}+\lambda_{3}\left(\epsilon_{H} E_{H}-\left(\mu_{H}+r_{H}+u_{1}(t)\right) I_{H}\right) \\
& +\lambda_{4}\left(b \beta_{V} S_{V} I_{H}-\left(\epsilon_{V}+\mu_{V}+u_{2}(t)\right) E_{V}\right)+\lambda_{5}\left\{\frac{M}{N_{V}}+\left(\epsilon_{V}-u_{2}(t)\right) E_{V}\right. \\
& \left.-\left(\mu_{V}+u_{2}(t)\right) I_{V}\right\} .
\end{aligned}
$$

The adjoint system is

$$
\begin{aligned}
& \frac{d \lambda_{1}}{d t}=-\lambda_{1}(t)\left(-\mu_{H}-\frac{b \beta_{H} N_{V} I_{V}}{N_{H}}\right)-\frac{b \beta_{H} N_{V}}{N_{H}} I_{V} \lambda_{2}(t), \\
& \frac{d \lambda_{2}}{d t}=-\lambda_{2}(t)\left(-\epsilon_{H}-\mu_{H}\right)-\lambda_{3}(t) \epsilon_{H}, \\
& \frac{d \lambda_{3}}{d t}=-\lambda_{3}(t)\left(-\mu_{H}-r_{H}-u_{1}^{*}\right)-b \beta_{V} S_{V} \lambda_{4}(t)-B_{0}, \\
& \frac{d \lambda_{4}}{d t}=-\lambda_{4}(t)\left(-\epsilon_{V}-\mu_{V}-u_{2}^{*}\right)-\lambda_{5}(t)\left(\epsilon_{V}-u_{2}^{*}\right), \\
& \frac{d \lambda_{5}}{d t}=\frac{b \beta_{H} S_{H} N_{V}}{N_{H}}\left(\lambda_{1}(t)-\lambda_{2}(t)\right)-\lambda_{5}(t)\left(-\mu_{V}-u_{2}^{*}\right) .
\end{aligned}
$$

Hence

$$
\begin{aligned}
& \frac{\partial H}{\partial u_{1}}=B_{1} u_{1}-\lambda_{3} I_{H}=0 \quad \Longrightarrow \quad u_{1}^{*}=\frac{\lambda_{3} I_{H}}{B_{1}}, \\
& \frac{\partial H}{\partial u_{2}}=B_{2} u_{2}-\lambda_{4} E_{V}+\lambda_{5}\left(-E_{V}-I_{V}\right)=0 \quad \Longrightarrow \quad u_{2}^{*}=\frac{\lambda_{4} E_{V}+\lambda_{5}\left(I_{V}+E_{V}\right)}{B_{2}} .
\end{aligned}
$$

Application of the property of the control set yields

$$
\begin{gathered}
u_{1}^{*}= \begin{cases}0 & \text { if } \frac{\lambda_{3} I_{H}}{B_{1}} \leq 0, \\
\frac{\lambda_{3} I_{H}}{B_{1}} & \text { if } \frac{\lambda_{3} I_{H}}{B_{1}}<u_{1}^{\max }, \\
u_{1}^{\max } & \text { if } \frac{\lambda_{3} I_{H}}{B_{1}} \geq u_{1}^{\max },\end{cases} \\
u_{2}^{*}= \begin{cases}0 & \text { if } \frac{\lambda_{4} E_{V}+\lambda_{5}\left(I_{V}+E_{V}\right)}{B_{2}} \leq 0, \\
\frac{\lambda_{4} E_{V}+\lambda_{5}\left(I_{V}+E_{V}\right)}{B_{2}} & \text { if } \frac{\lambda_{4} E_{V}+\lambda_{5}\left(I_{V}+E_{V}\right)}{B_{2}}<u_{2}^{\max }, \\
u_{2}^{\max } & \text { if } \frac{\lambda_{4} E_{V}+\lambda_{5}\left(I_{V}+E_{V}\right)}{B_{2}} \geq u_{2}^{\max } .\end{cases}
\end{gathered}
$$


Table 2 Parameters used in the numerical simulations

\begin{tabular}{lll}
\hline Parameter & Case 1 & Case 2 \\
\hline$\mu_{H}$ & $1 /(70 * 365)$ & $1 /(70 * 365)$ \\
$N_{H}$ & 92,000 & 92,000 \\
$b$ & 0.2 & 0.2 \\
$A$ & 5000 & 5000 \\
$\mu_{V}$ & $1 / 24$ & $1 / 24$ \\
$M$ & 400 & 0 \\
$\beta_{H}$ & 0.95 & 0.95 \\
$\beta_{V}$ & 0.75 & 0.75 \\
$\epsilon_{H}$ & 0.1667 & 0.1667 \\
$\epsilon_{V}$ & 0.1428 & 0.1428 \\
$r_{H}$ & 0.3 & 0.3 \\
\hline
\end{tabular}

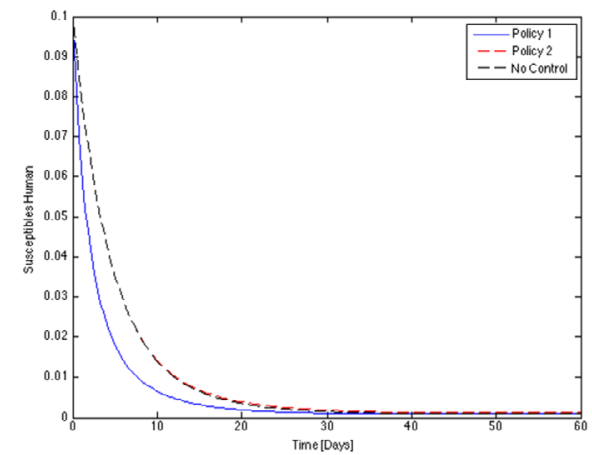

(a) $S_{H}$ individuals of case 1

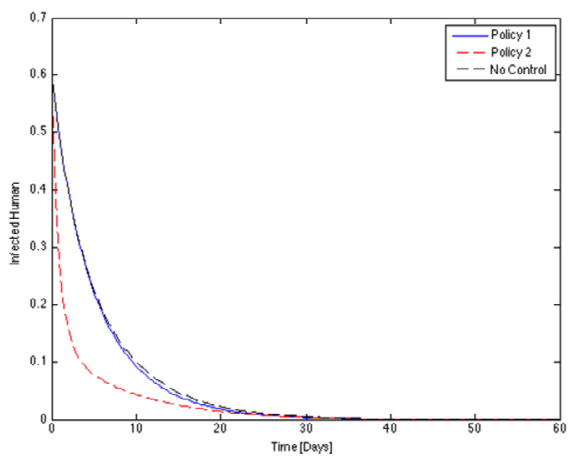

(c) $I_{H}$ individuals of case 1

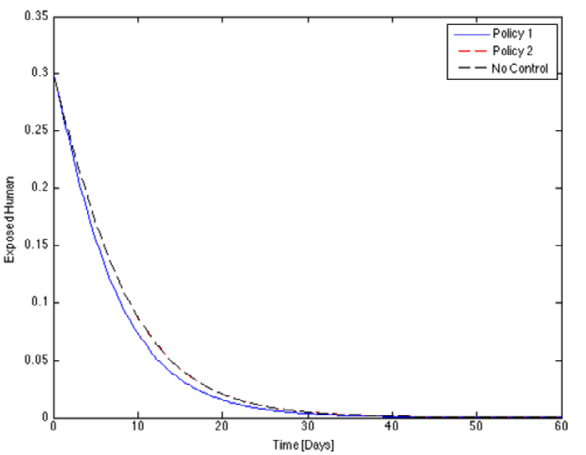

(b) $E_{H}$ individuals of case 1

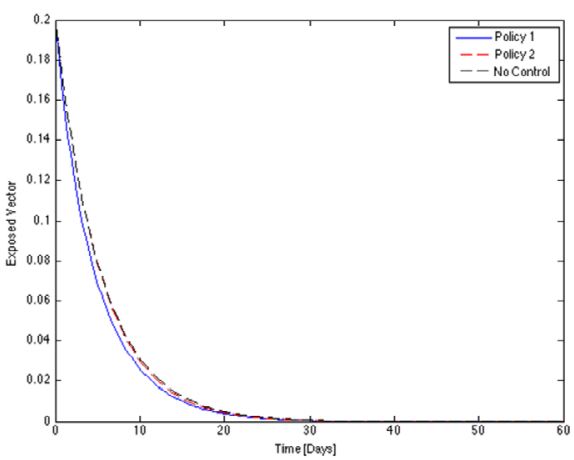

(d) $E_{V}$ individuals of case 1

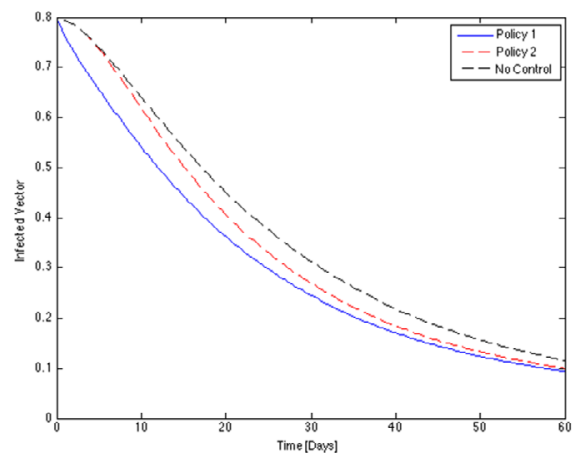

(e) $I_{V}$ individuals of case 1

Figure 1 The responses comparisons of case 1 


\section{Results and discussion}

In this section we give the numerical analyses of the two control policies in containing the dengue outbreak. For each policy, the optimality system is numerically solved using the fourth order Runge-Kutta forward-backward sweep method [32]. Specifically, the differential equation systems of Equations (29)-(33) and (34)-(38) are solved by the forward Runge-Kutta method with the predefined initial conditions, while the adjoint system is solved by the backward sweep method with the transversality conditions. The parameters used are taken from Chanprasopchai et al. [19] and are given in Table 2.

The initial conditions used for all simulations are given as follows:

$$
\begin{array}{ll}
S_{H}(0)=0.1, \quad E_{H}(0)=0.3, \quad I_{H}(0)=1-S_{H}(0)-E_{H}(0)=0.6, \\
E_{V}(0)=0.2, \quad I_{V}(0)=1-E_{V}(0)=0.8 .
\end{array}
$$

The time $T$ used for all simulations is fixed to 60 days or two months which is around the average infection season duration. The values of the control weights $B_{0}, B_{1}$ and $B_{2}$ are set initially at $B_{0}=100, B_{1}=50, B_{2}=200$. Note that the cost of operating the pesticide control $B_{2}$ is set higher than the costs of vaccination and isolation, since insecticide control is generally more labor intensive and time consuming to implement than both vaccination and isolation.

Figure 1 shows the scenario of controlling the dengue transmission subjected to the vertical transmission, where the controls of Policies 1 and 2 are applied to the system (case 1). Note that in practice both controlling mechanisms $u_{1}$ and $u_{2}$ cannot be implemented over the entire population, and therefore the maximum level of control $u_{\max }$ is kept at 0.8 for

Figure 2 The control comparisons for case 1

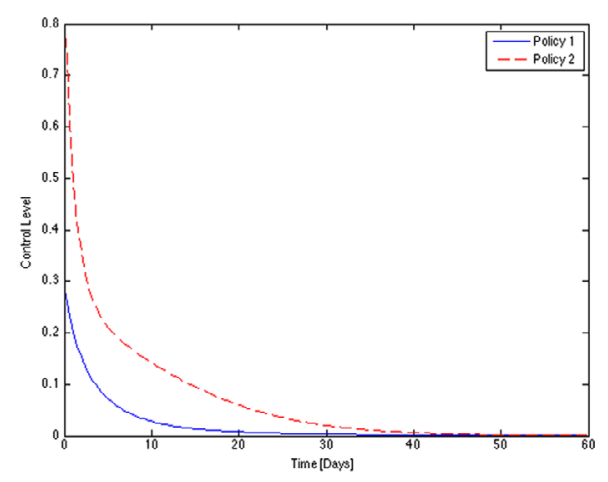

(a) The control $u_{1}$ for case 1

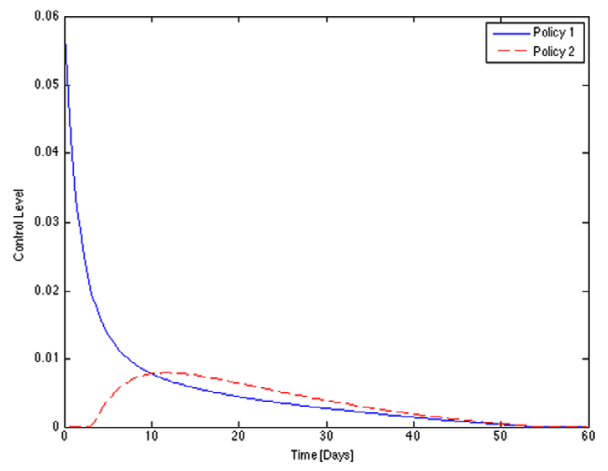

(b) The control $u_{2}$ for case 1 
both $u_{1}$ and $u_{2}$ in both policies. It is seen from Figs. 1(a) and 1(b) that the number of individuals with susceptibility to the disease $S_{H}$ and the number of exposed individuals $E_{V}$ significantly deviate from the uncontrolled system upon the action of Policy 1 ; whereas Policy 2 yields similar responses for $S_{H}$ and $E_{H}$. However, the number of infected individuals $I_{H}$, shown in Fig. 1(c), is lowest upon the action of Policy 2; whereas the action of Policy 1 yields only a marginally improved response to the uncontrolled system. Figures $1(\mathrm{~d})$ and 1 (e) show the responses of the numbers of the exposed and infected vector populations. It is evident that the number of infected vector populations under the action of Policy 1 is significantly less than that of Policy 2 .

Figure 2 shows the expended control actions of $u_{1}$ and $u_{2}$ to control the dengue transmission when vertical transmission is possible. It is seen that for $u_{1}$ both policies require

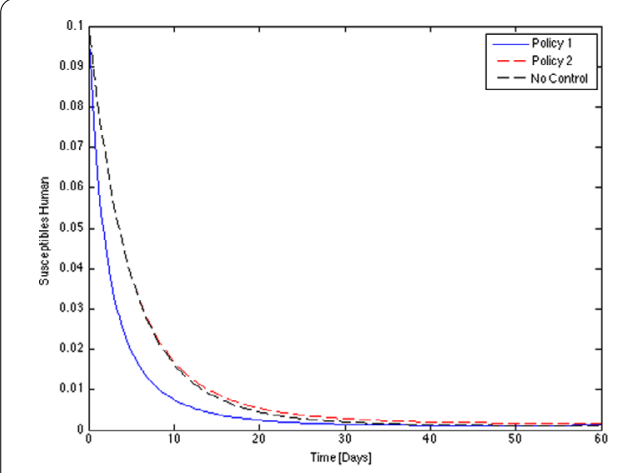

(a) $S_{H}$ individuals of case 2

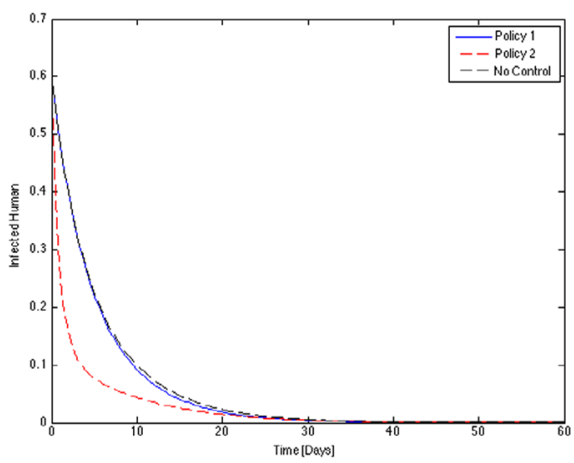

(c) $I_{H}$ individuals of case 2

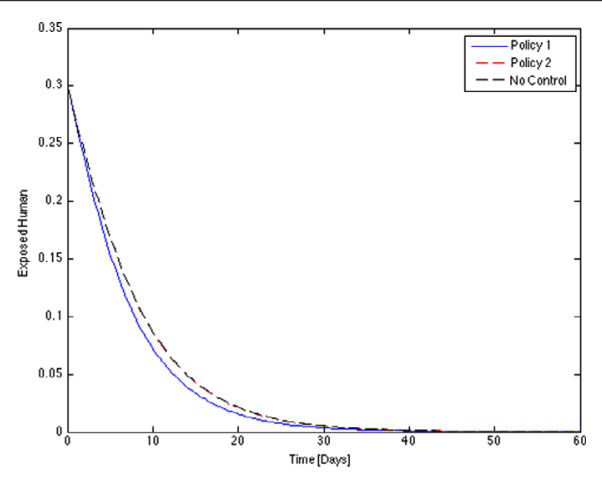

(b) $E_{H}$ individuals of case 2

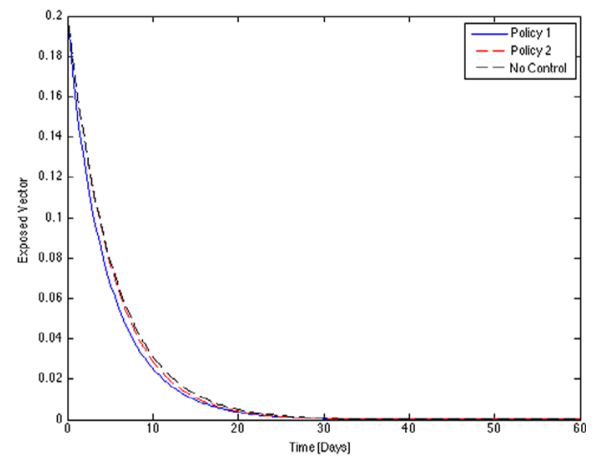

(d) $E_{V}$ individuals of case 2

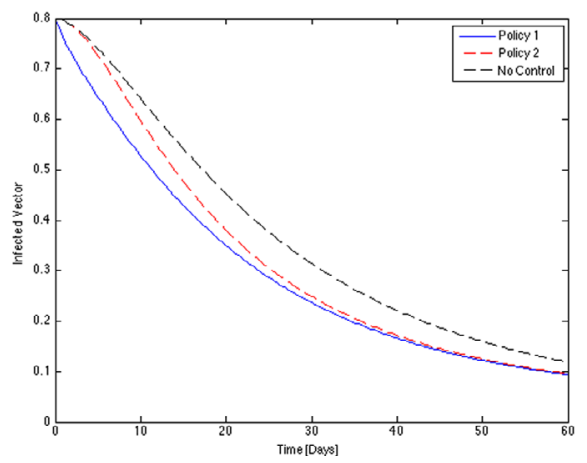

(e) $I_{V}$ individuals of case 2

Figure 3 The responses comparisons of case 2 
a control that starts at some starting point, which then quickly decays to zero. For $u_{2}$, Policy 1 expends a small control action that quickly decays to zero, while Policy 2 requires zero control initially, which then quickly increases initially before decaying to zero. Note that this increase happens around Day 8, which coincides with the sharp drop of the infected human individuals. Although Policy 2 leads to a quicker drop of the infected human population, this comes with a far greater initial isolation effort, whereas the required vaccination effort is significantly less while yielding an improved all-round epidemic control.

Figure 3 shows the effects of applying the control measures to the dengue transmission when there is no vertical transmission (case 2). Note that in this case all the parameters of case 1 were kept the same, except that now $M=0$. It is evident from Figs. 3(a) and 3(b) that once again the number of susceptible human individuals and the number of exposed individuals decay quicker to a level close to zero under the action of Policy 1, while the number of the infected human populations drops quicker to a level close to zero when the controls of Policy 2 are applied. The implementation of Policy 1 initially yields a lower $I_{V}$ response than that of Policy 2 in the first 30 days. The two responses converge to the same number thereafter. This is in contrast to case 1 , where this convergence is not seen until Day 57. This result suggests that the control is more enhanced for case 2 in favor of case 1 , to facilitate a quicker drop in the $I_{V}$ individuals.

Figure 4 shows the inputs $u_{1}$ and $u_{2}$ needed to control the spread of the dengue disease. It is seen that similar levels of $u_{1}$ in comparison to case 1 were needed in both policies to control the disease; whereas the level of the insecticide administration $u_{2}$ is slightly higher in the presence of vertical transmission. This greater $u_{2}$ is to be anticipated because the presence of vertical transmission introduces transovarially infected mosquitoes into

Figure 4 The control comparisons for case 1

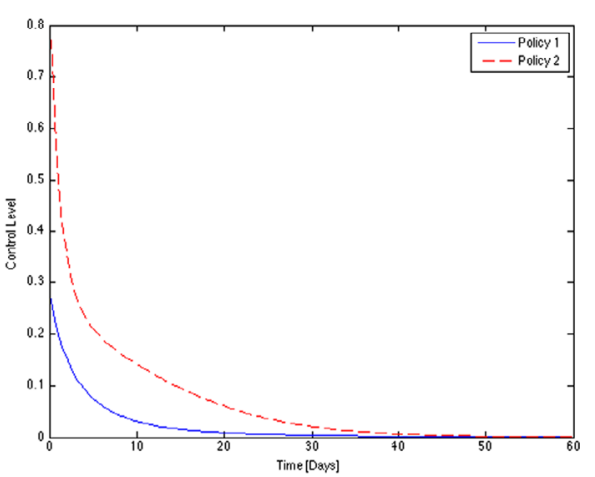

(a) The control $u_{1}$ for case 1

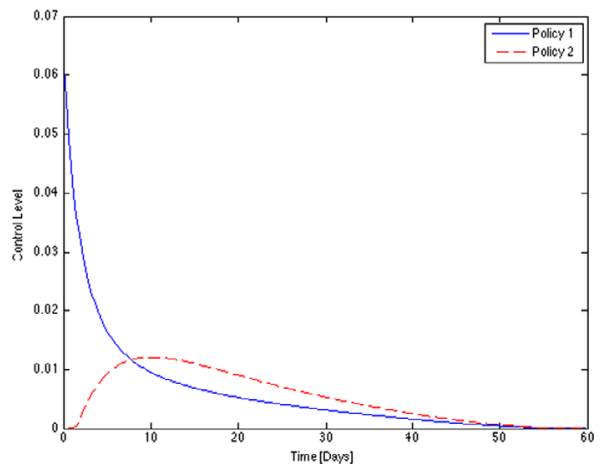

(b) The control $u_{2}$ for case 1 
the system, thereby requiring higher insecticide administration to facilitate the control so that the epidemic achieves the same state quicker than the case without the vertical transmission.

\subsection{Change in $B_{0}$}

To investigate the controlled system responses upon respective weight changes in the objective functional, the parameter $B_{0}$ is firstly chosen to be investigated. To this end, the parameter $B_{0}$, which is associated with the human infectious population, is firstly defined as follows:

$$
B_{0}=[200,400, \ldots, 1000] .
$$

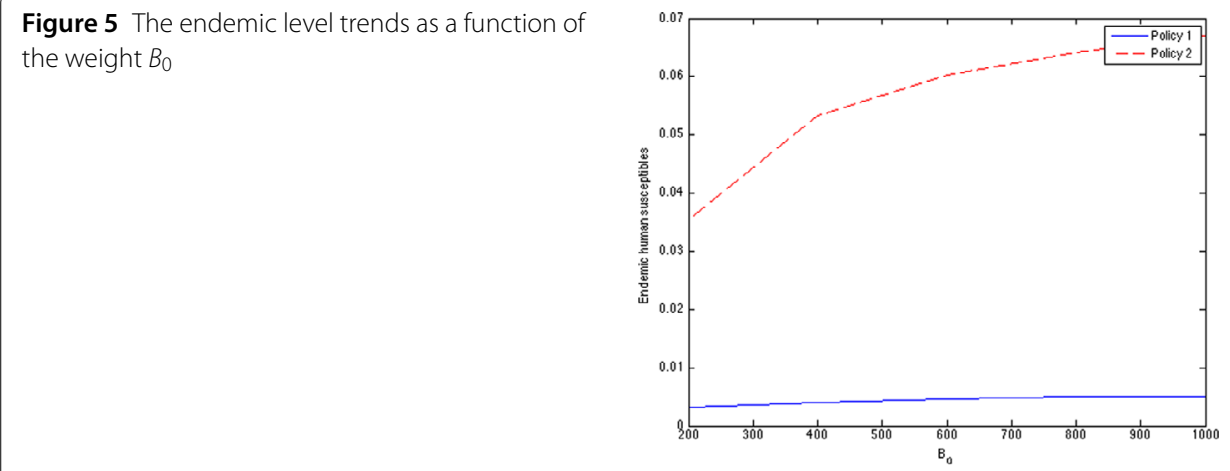

(a) $S_{H}^{*}$ trend as a function of the weight $B_{0}$

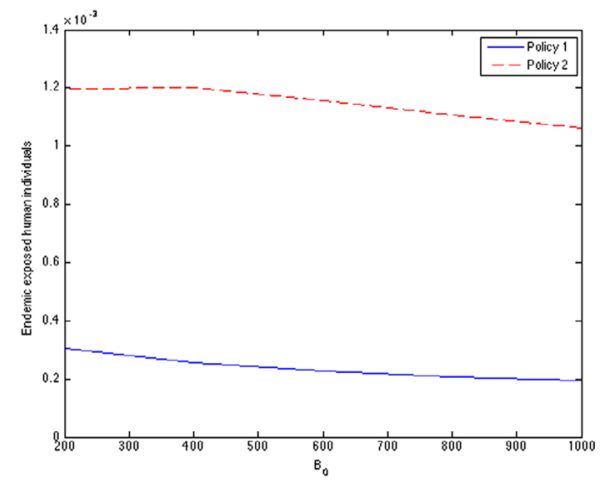

(b) $E_{H}^{*}$ trend as a function of the weight $B_{0}$

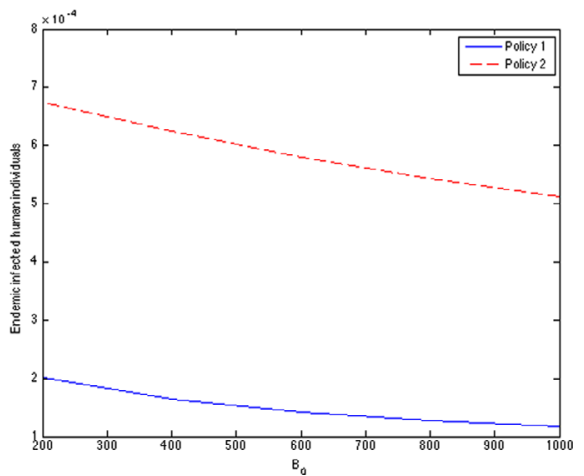

(c) $I_{H}^{*}$ trend as a function of the weight $B_{0}$ 
The other epidemiological parameters are chosen from case 2 of Table 2 . The weight $B_{1}$ is kept fixed at 200, while the weight $B_{2}$ is fixed at 400 for all simulations.

Figure 5 plots the changes of the endemic level trends as a function of the weight $B_{0}$. It is evident from Fig. 5(a) that for both policies, the $S_{H}^{*}$ trends result in an increasing function with decreasing gradient as the weight $B_{0}$ increases. Figures 5(b) and 5(c) illustrate that for both policies, the $E_{H}^{*}$ and $I_{H}^{*}$ trends yield a decreasing function with negative $\frac{d E^{*}}{d B_{0}}$ and $\frac{d I^{*}}{d B_{0}}$. Specifically, these graphs show that a higher $B_{0}$ weighting gives rise to a lower endemic exposed human individuals, as well as a lower endemic infectious human individuals.

Figure 6 now plots the control efforts of both controlling actions $u_{1}$ and $u_{2}$, which are used to implement Policies 1 and 2, along the change in $B_{0}$ of Equation (68). It is seen from Figs. 6(a) and 6(c) that for $u_{1}$ and $u_{2}$ used to implement Policy 1 , a higher $B_{0}$ weighting requires higher initial values of $u_{1}(t)$ and $u_{2}(t)$ in order to better control the disease. Figure 6 (b) shows that as $B_{0}$ increases, the $u_{1}$ action stays at the maximum $u_{1, \max }$ for a longer period of time before decaying to zero. Figure $6(\mathrm{~d})$ shows the higher value of $B_{0}$, the higher initial value of $u_{2}(t)$, as well as a the higher value of the saddle which exists around Day 15-30, to better control the dengue transmission effectively. It is thus our recommendation that a higher $B_{0}$ weighting should be set to ensure a better dengue disease control when implementing either Policy 1 or Policy 2.

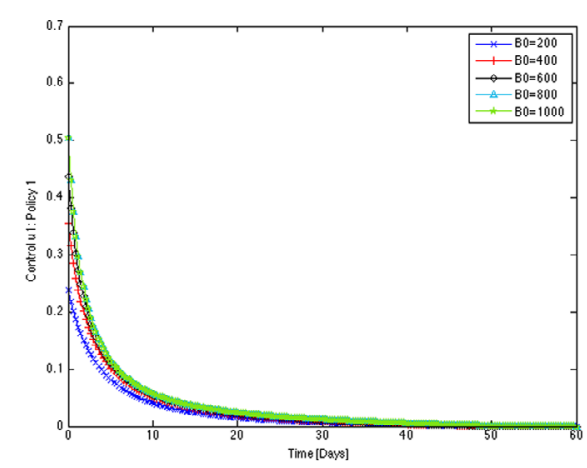

(a) The comparison of $u_{1}$ policy 1 actions along the change in $B_{0}$.

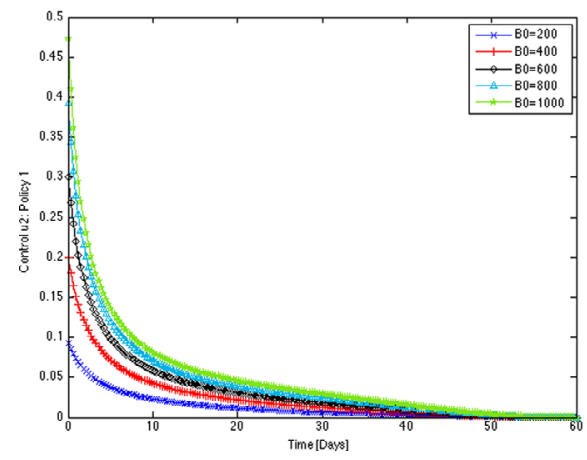

(c) The comparison of $u_{2}$ policy 1 actions along the change in $B_{0}$.

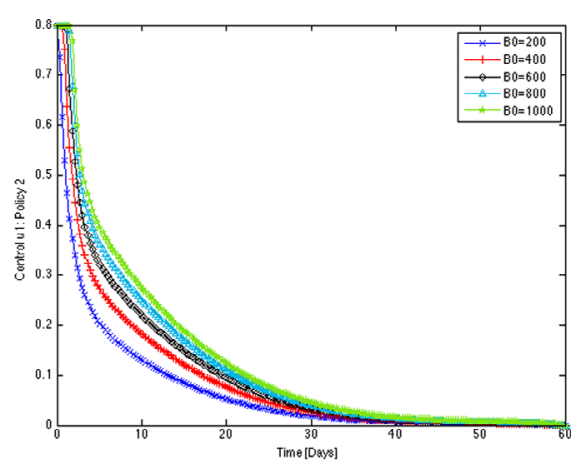

(b) The comparison of $u_{1}$ policy 2 actions along the change in $B_{0}$.

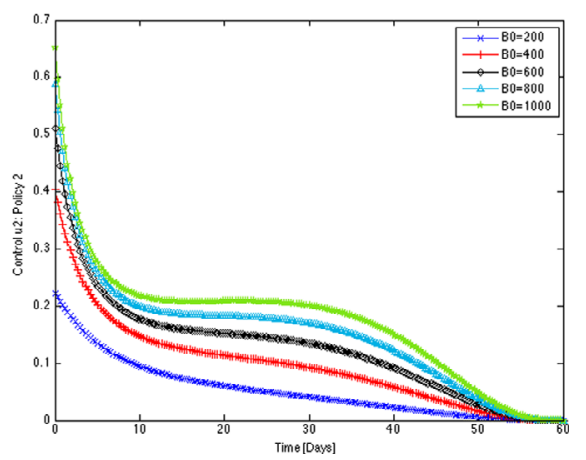

(d) The comparison of $u_{2}$ policy 2 actions along the change in $B_{0}$.

Figure 6 The comparison of the control actions along the change in $B_{0}$ 


\subsection{Change in $B_{1}$}

We now turn our attention to investigating controlled system responses to the change in the weight $B_{1}$. To this end, the $B_{1}$ vector is defined as follows:

$$
B_{1}=[200,400, \ldots, 1000] .
$$

The other epidemiological parameters are again chosen from case 2 of Table 2 . The weight $B_{0}$ is fixed at 1000 , while $B_{2}$ is fixed at 400 .

Figure 7 now plots the endemic level changes as a function of $B_{1}$. Figures $7(a)-7(\mathrm{c})$ show that the endemic levels of $S_{H}^{*}, E_{H}^{*}$, and $I_{H}^{*}$ give an increasing function with increasing gradient as $B_{1}$ increases for both Policies 1 and 2. Specifically, these graphs suggest that a lower $B_{1}$ weighing is favorable to yield the lowest numbers of susceptible, infectious, and exposed human individuals.

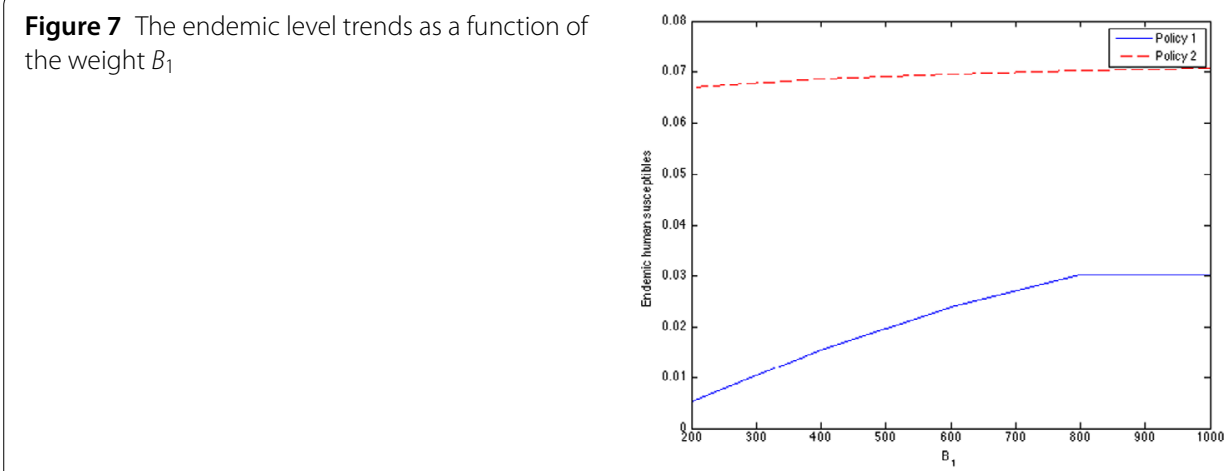

(a) $S_{H}^{*}$ trend as a function of the weight $B_{1}$

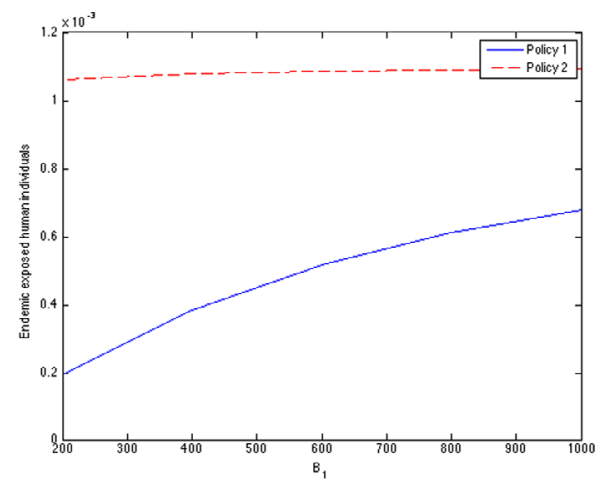

(b) $E_{H}^{*}$ trend as a function of the weight $B_{1}$

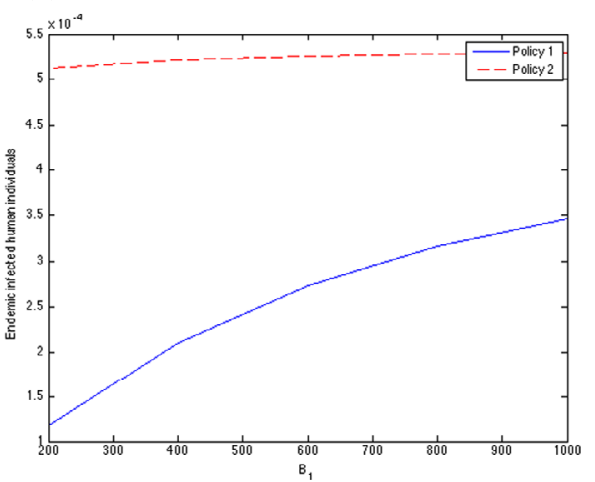

(c) $I_{H}^{*}$ trend as a function of the weight $B_{1}$ 


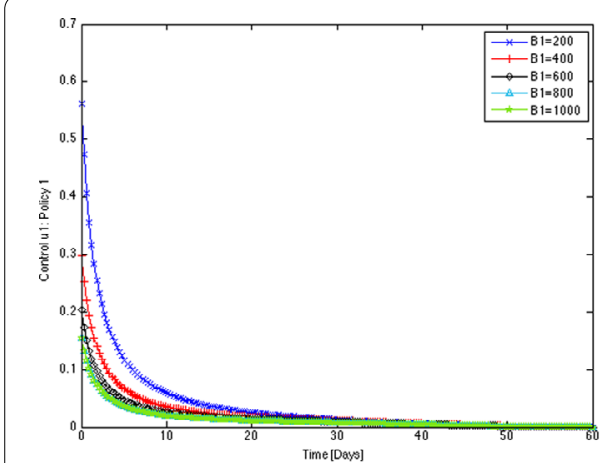

(a) The comparison of $u_{1}$ policy 1 actions along the change in $B_{1}$.

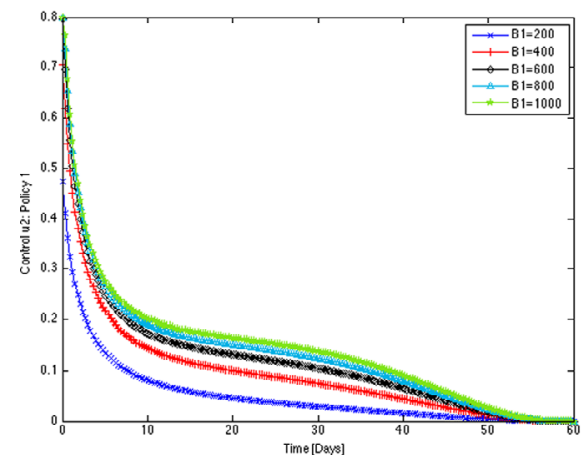

(c) The comparison of $u_{2}$ policy 1 actions along the change in $B_{1}$.

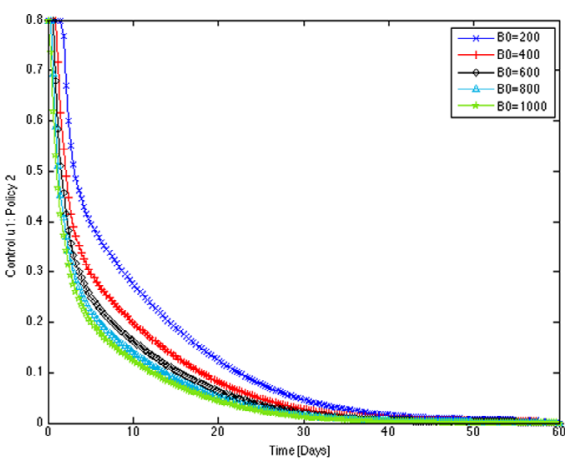

(b) The comparison of $u_{1}$ policy 2 actions along the change in $B_{1}$.

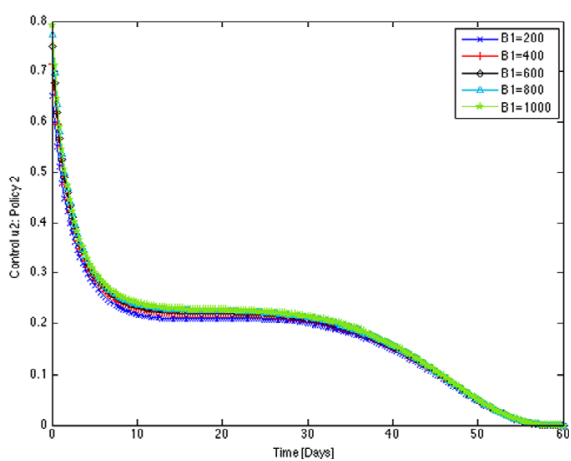

(d) The comparison of $u_{2}$ policy 2 actions along the change in $B_{1}$.

Figure 8 The comparison of the control actions along the change in $B_{1}$

Figure 8 plots the control efforts for both controlling actions used to implement Policies 1 and 2 against the change of the weighting function $B_{1}$. It is seen from Fig. 8(a) that the lower the $B_{1}$ weighting, the higher initial $u_{1}(t)$ is needed to implement Policy 1 . Figure $8(\mathrm{~b})$ illustrates that the lower the $B_{1}$ weighting, the longer $u_{1}$ action remains at the maximum $u_{1, \max }$ before decaying. Figures $8(\mathrm{c})$ and $8(\mathrm{~d})$ suggest that a higher $B_{1}$ weighting results in more control effort $u_{2}(t)$ for both policies. Hence it is advisable to set a lower $B_{1}$ weighting to minimize the endemic response levels as well as minimizing the required control efforts to effective control the dengue.

\subsection{Change in $B_{2}$}

To investigate the controlled system responses to changes in the weight $B_{2}$, let us now define the $B_{2}$ vector by

$$
B_{2}=[200,400, \ldots, 1000] .
$$

The other epidemiological constants are kept identical to those used to investigate the changes in $B_{0}$ and $B_{1}$. The weights $B_{0}$ and $B_{1}$ were fixed at 1000 and 400 respectively.

The result of Fig. 9(a) suggests that for both policies, the endemic levels of $S_{H}^{*}$ decrease as the weighting function $B_{2}$ increases. Figures 9(b) and 9(c) suggest that the endemic levels 
Figure 9 The endemic level trends as a function of the weight $B_{2}$

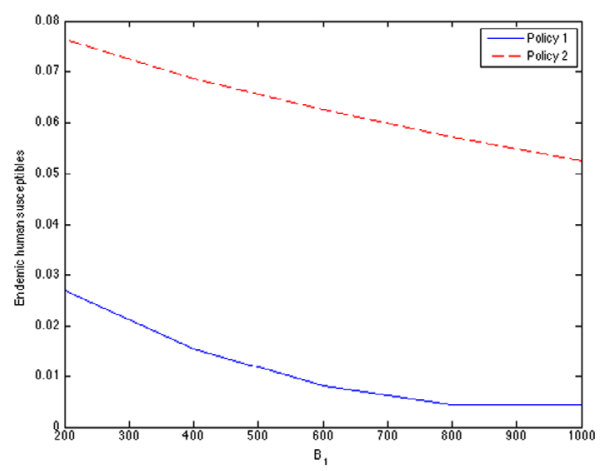

(a) $S_{H}^{*}$ trend as a function of the weight $B_{2}$

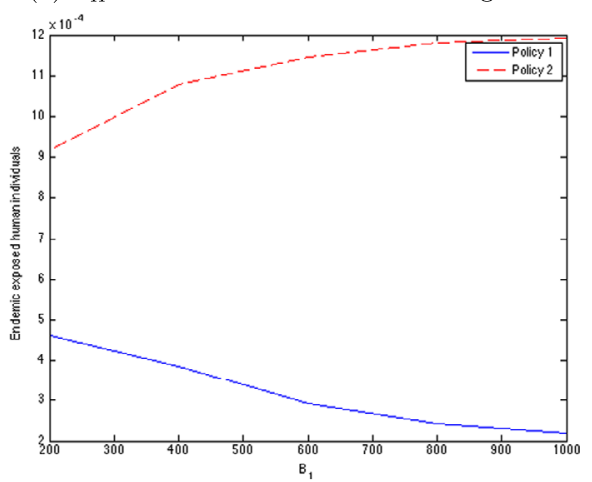

(b) $E_{H}^{*}$ trend as a function of the weight $B_{2}$

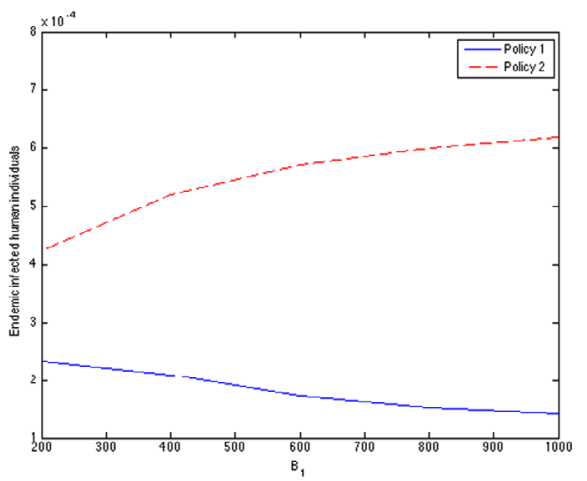

(c) $I_{H}^{*}$ trend as a function of the weight $B_{2}$

$E_{H}^{*}$ and $I_{H}^{*}$ will be decreasing when the action of Policy 1 is carried out but will increase if the actions of Policy 2 are performed. These results suggest that for the administration of Policy 1, a higher $B_{2}$ would yield a more favorable outcome in terms of minimizing the number of infectious and exposed humans, while for the administration of Policy 2, a lower $B_{2}$ would better minimize the number of infectious and exposed individuals.

Figure 10(a) suggests that the lower the $B_{2}$ weighting, the higher the initial $u_{1}(t)$ required to implement Policy 1 ; changing $B_{2}$ however does not significantly alter the $u_{1}$ needed to implement Policy 2, unlike in the case of Policy 1. Figures 10(c) and 10(d) suggest that a lower $B_{2}$ will result in more control efforts $u_{2}$ being expended to implement both policies. Overall it is advisable to set a low $B_{2}$ weighting to give an effective control of the dengue disease. 


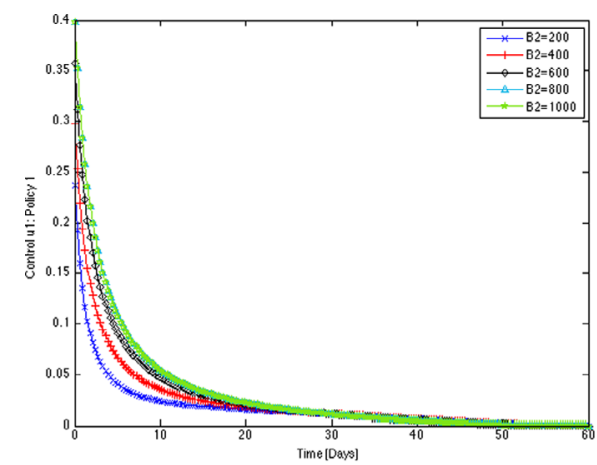

(a) The comparison of $u_{1}$ policy 1 actions along the change in $B_{2}$.

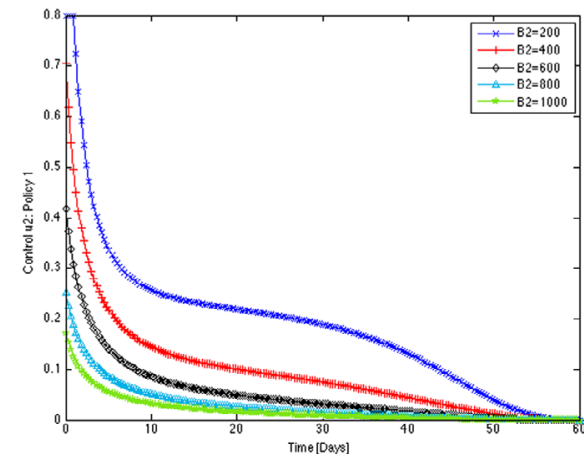

(c) The comparison of $u_{2}$ policy 1 actions along the change in $B_{2}$.

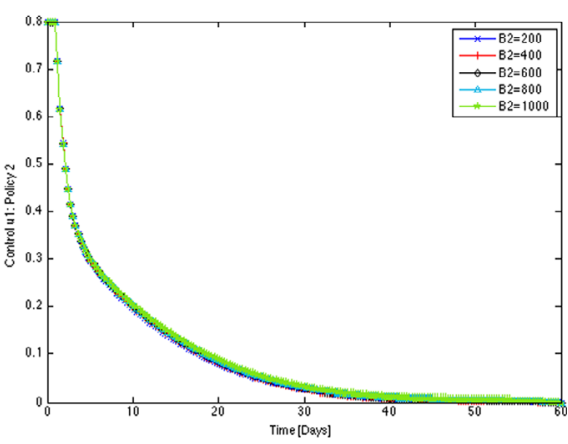

(b) The comparison of $u_{1}$ policy 2 actions along the change in $B_{2}$.

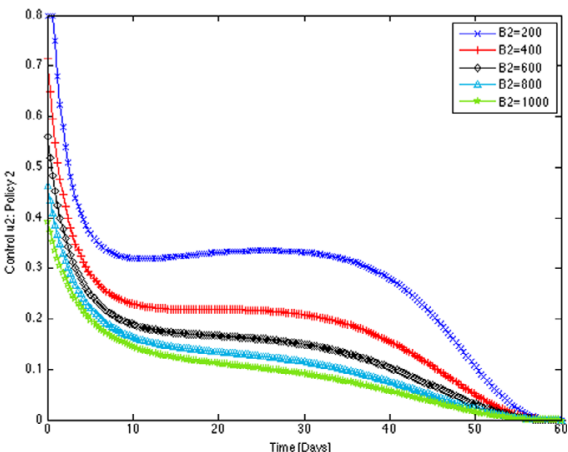

(d) The comparison of $u_{2}$ policy 2 actions along the change in $B_{2}$.

Figure 10 The comparison of the control actions along the change in $B_{2}$

\subsection{Changes in $\beta_{H}$}

To investigate the control effort needed to withstand the changes in the transmission probability of the dengue virus from vector to human, let us define the $\beta_{H}$ vector by

$$
\beta_{H}=[0.65,0.75,0.85,0.95]
$$

The other epidemiological parameters are kept identical to the earlier investigations. The weights $B_{0}, B_{1}$, and $B_{2}$ are fixed at 1000,400 , and 800 respectively.

Figures 11(a) and 11(b) suggest that as the transmission probability from vector to human increases, the $u_{1}$ control for Policy 1 begins at an initial value, then gradually decays to zero effort. The required $u_{1}$ control for Policy 2 stays at the maximum value $u_{1 \text {,max }}$ for around three days, then gradually decays to zero. Nevertheless, there is little deviation between the $u_{1}$ control efforts as $\beta_{H}$ increases. Figures 11(c) and 11(d) suggest that for Policy 1, a lower $\beta_{H}$ required a lower control effort, whereas for Policy 2, a higher $\beta_{H}$ required a little higher control efforts around Day 20. However, these differences in the required control efforts are mainly within $2 \%$ of one another, thereby suggesting that the both control schemes are robust to changes in $\beta_{H}$. 


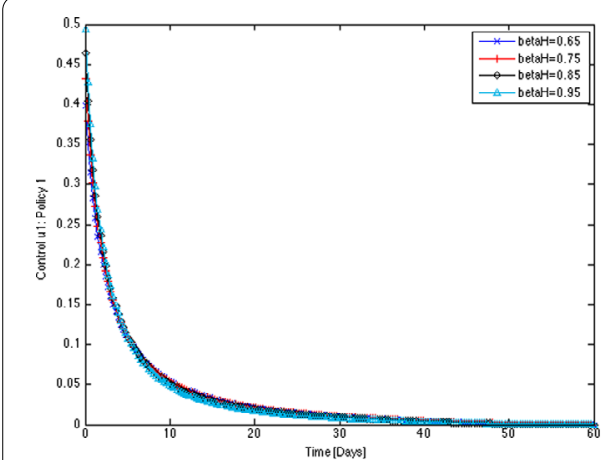

(a) The comparison of $u_{1}$ policy 1 actions along the change in $\beta_{H}$.

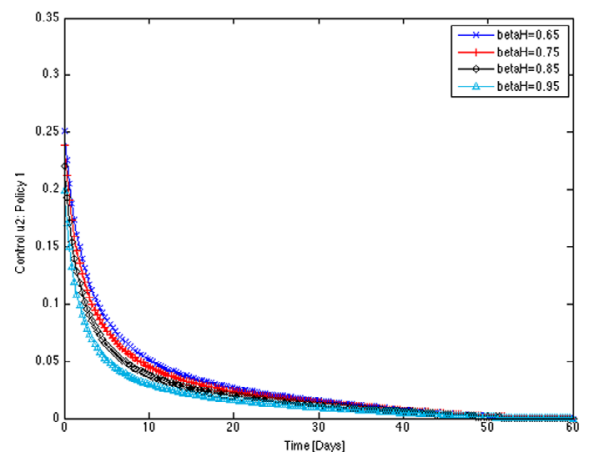

(c) The comparison of $u_{2}$ policy 1 actions along the change in $\beta_{H}$.

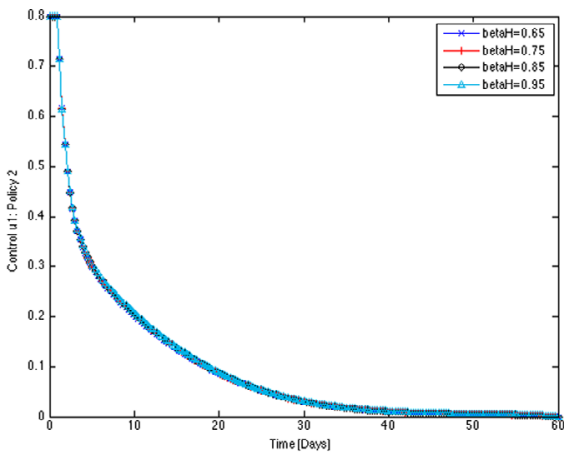

(b) The comparison of $u_{1}$ policy 2 actions along the change in $\beta_{H}$.

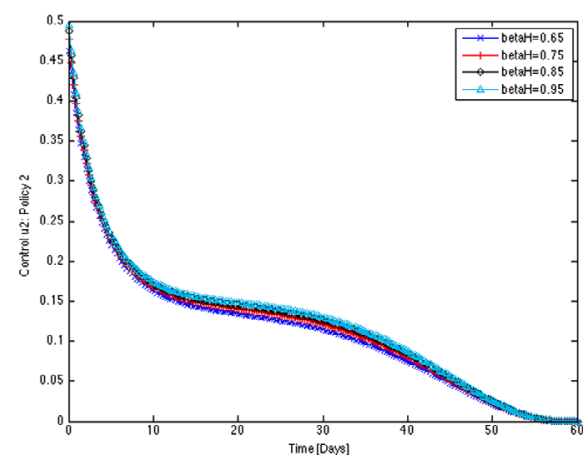

(d) The comparison of $u_{2}$ policy 2 actions along the change in $\beta_{H}$.

Figure 11 The comparison of the control actions along the change in $\beta_{H}$

\subsection{Changes in $\beta_{V}$}

To investigate the control effort needed to withstand the changes in the transmission probability of the dengue virus from human to vector, let us now define the $\beta_{V}$ vector by

$$
\beta_{V}=[0.65,0.75,0.85,0.95] .
$$

The other epidemiological parameters are kept identical to the earlier investigations. The weights $B_{0}, B_{1}$, and $B_{2}$ are fixed at 1000, 200, and 400 respectively.

Figure 12 shows that, overall, there are only subtle differences between the required $u_{1}(t)$ and $u_{2}(t)$ needed to implement both policies, as the values of $\beta_{V}$ increase from 0.65 to 0.95 . These results suggest that both controlling schemes are also robust to the changes in the transmission rate between vector to human $\beta_{V}$.

\section{Conclusion}

This work has presented a control mechanism based on a previously developed mathematical model of the dengue disease by Chanprasopchai et al. that takes into account the effect of vertical transmission [19]. The optimal control framework was proposed in view of two policies, namely vaccination and insecticide administration (Policy 1) and isolation and insecticide administration (Policy 2). The use of Pontrargin's maximum principle allowed necessary and optimality conditions, thus facilitating the optimal control to be developed. 


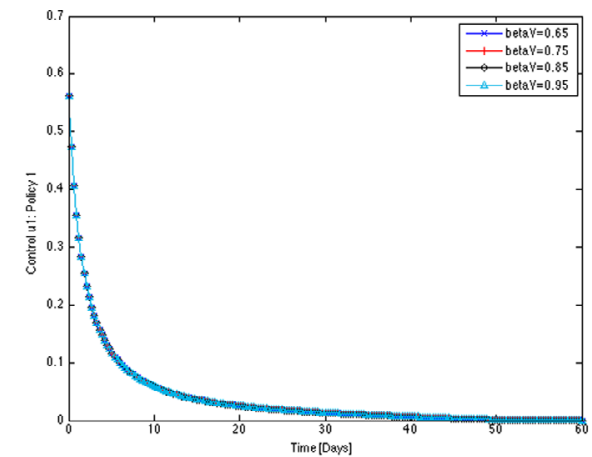

(a) The comparison of $u_{1}$ policy 1 actions along the change in $\beta_{V}$.

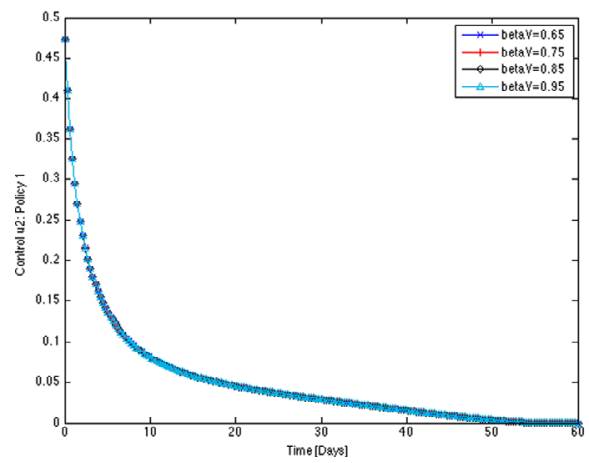

(c) The comparison of $u_{2}$ policy 1 actions along the change in $\beta_{V}$.

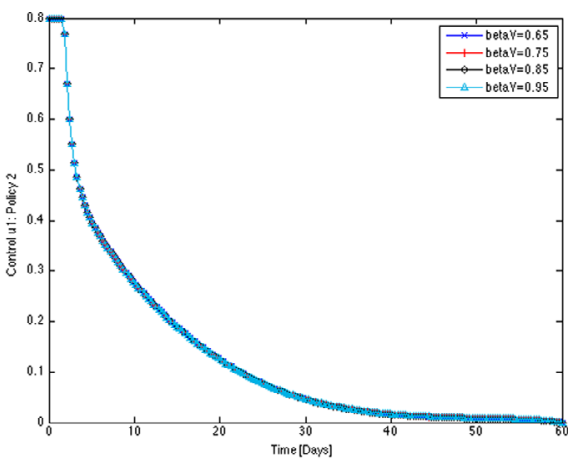

(b) The comparison of $u_{1}$ policy 2 actions along the change in $\beta_{V}$.

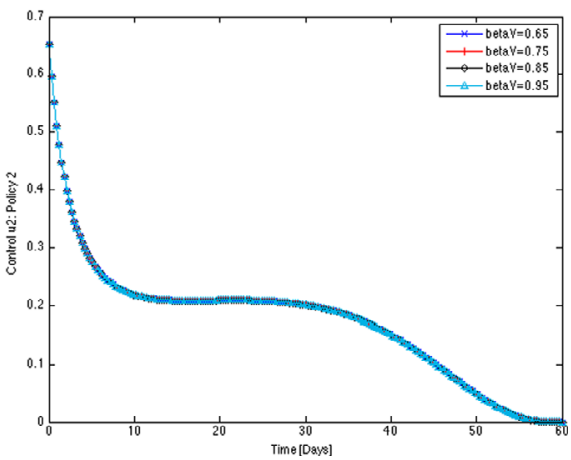

(d) The comparison of $u_{2}$ policy 2 actions along the change in $\beta_{V}$.

Figure 12 The comparison of the control actions along the change in $\beta_{V}$

Numerical solutions of the control systems were presented. It was found that, although the administration of Policy 2 yielded a quicker diminishment of the infected human population, this comes with a greater expense in the initial effort; whereas the required vaccination effort of Policy 1 is significantly less, while yielding an improved all-round epidemic control. Investigations were also conducted to investigate the control systems response under the changes of the weight functions $B_{0}, B_{1}$, and $B_{2}$. Numerical results suggest that the endemic levels $E_{H}^{*}$ and $I_{H}^{*}$ generally yielded a decreasing trend for both administered policies with higher $B_{0}$; whereas an increasing trend is seen for both administered policies with higher $B_{1}$. For $B_{2}$, an increasing function is obtained for the implementation of Policy 1 , while a decreasing function is attained for Policy 2 . These results also suggest that a high $B_{0}$ weighting, along with low $B_{1}$ and $B_{2}$ values, ensured the minimization of the endemic response levels, as well as minimizing the control efforts to control the dengue.

Lastly, the two control schemes are shown numerically to be robust to changes in the transmission probabilities, both $\beta_{H}$ and $\beta_{V}$. Hence, besides using insecticides, vaccinations and isolation also help to effectively and optimally control the dengue disease.

Acknowledgements

Not applicable.

Funding

Not applicable. 
Availability of data and materials

The dataset generated and used to support the findings of this work are available from the corresponding author upon reasonable request.

\section{Ethics approval and consent to participate}

Not applicable.

\section{Competing interests}

The authors declare that there are no conflicts of interest regarding the publication of this paper.

\section{Consent for publication}

All authors are in consensus for the publication of this manuscript.

\section{Authors' contributions}

PP provided the mathematical model of the dengue fever disease, set up the optimal control problem, and wrote half of the manuscript. NW suggested the use of two policies, conducted the numerical analyses of the control, and wrote half of the manuscript. IMT improved greatly the manuscript. All authors read and approved the final version of the manuscript.

\section{Author details}

'Department of Mathematics, Faculty of Science, King Mongkut's Institute of Technology Ladkrabang, Bangkok, Thailand. ${ }^{2}$ Computational \& Applied Science for Smart Innovation Cluster (CLASSIC), Department of Mathematics, Faculty of Science, King Mongkut's University of Technology Thonburi, Bangkok, Thailand. ${ }^{3}$ Department of Instrumentation and Control Engineering, Faculty of Engineering, King Mongkut's Institute of Technology Ladkrabang, Bangkok, Thailand.

\section{Publisher's Note}

Springer Nature remains neutral with regard to jurisdictional claims in published maps and institutional affiliations.

Received: 1 February 2019 Accepted: 25 April 2019 Published online: 09 May 2019

\section{References}

1. Gubler, D.J.: Dengue and dengue hemorrhagic fever. Clin. Microbiol. Rev. 11, 480-496 (1998)

2. World Health Organization: Dengue and severe dengue. Dengue Situation Update Number 554 (2018). Accessed 29 Jan 2019

3. Reich, N., et al.: Interactions between serotypes of dengue highlight epidemiological impact of cross-immunity. J. R. Soc. Interface 10(86), 20130414 (2013)

4. Cavalli-Sforza, L.L., Feldman, M.: Cultural Transmission and Evolution: A Quantitative Approach. Princeton University Press, New Jersey (1981)

5. Rosen, L.: Sexual transmission of dengue viruses by Aedes albopictus. Am. J. Trop. Med. Hyg. 37(2), 398-402 (1987)

6. Guenther, J., et al.: Evidence of vertical transmission of dengue virus in two endemic localities in the state of Oaxaca, Mexico. Intervirology 50(5), 347-352 (1987)

7. Sanchez-Vargas, I., et al.: Demonstration of efficient vertical and venereal transmission of denque virus type- 2 in a genetically diverse laboratory strain of Aedes aegypti. PLoS Negl. Trop. Dis. 12(8), e0006754 (2018)

8. Clements, A.N.: The egg shell. In: The Biology of Mosquitoes, pp. 63-73. CABI, Wallingford (2000)

9. Hadinegoro, R., et al.: Efficacy and long term safety of a dengue vaccine in regions of endemic disease. N. Engl. J. Med. 337, 1195-1206 (2015)

10. Aguiar, M., Stollenwerk, N., Halstead, H.B.: The impact of the newly licensed dengue vaccine in endemic countries. PLoS Negl. Trop. Dis. 10(12), e0005179 (2016)

11. Owen, D.: Philippines halts dengue immunisation campaign owing to safety risk. BMJ 359, j5759 (2017)

12. World Health Organization: Weekly Epidemiological Record 93(36), 457-476 (2018)

13. Calain, P., Poncin, M.: Reaching out to Ebola victims: coercion, persuasion or an appeal for self-sacrifice? Soc. Sci. Med. $147,126-133(2015)$

14. Derouich, M., Boutayeb, A.: Dengue fever: mathematical modelling and computer simulation. Appl. Math. Comput. $177(2), 528-544(2006)$

15. Esteva, L., Vargas, C.: Analysis of a dengue disease transmission model. Math. Biosci. 150(2), 131-151 (1998)

16. Esteva, L., Vargas, C.: A model for dengue disease with variable human population. J. Math. Biol. 38(3), 220-240 (1999)

17. Anggriani, N., et al.: The effect of reinfection with the same serotype on dengue transmission dynamics. Appl. Math. Comput. 349, 62-80 (2019)

18. Sardar, T., Rana, S., Chattopadhyay, J.: A mathematical model of dengue transmission with memory. Commun. Nonlinear Sci. Numer. Simul. 22, 511-525 (2015)

19. Chanprasopchai, P., Tang, I.M., Pongsumpun, P.: The SEIR dynamical transmission model of dengue disease with and without the vertical transmission of the virus. Am. J. Appl. Sci. 14(12), 1123-1145 (2017)

20. Yang, C.X., Nie, L.F.: The effect of vector control strategy against dengue transmission between mosquitoes and human. Electron. J. Qual. Theory Differ. Equ. 2017, 17 (2017)

21. Al-Sulami, H., et al.: On fractional order dengue epidemic model. Math. Probl. Eng. 2014, 456337 (2014)

22. Iboi, E., Gumel, A.B.: Mathematical assessment of the role of Dengraxia vaccine on the transmission dynamics of dengue serotypes. Math. Biosci. 304, 25-47 (2018)

23. Atangana, A., Goufo, E.F.D.: On the mathematical analysis of Ebola hemorrhagic fever: deathly infection disease in West African countries. BioMed Res. Int. 2014, 261383 (2014)

24. Atangana, A.: A novel model for the lassa hemorrhagic fever: deathly disease for pregnant women. Neural Comput. Appl. 26(8), 1895-1903 (2015) 
25. Atangana, A., Alkahtani, B.S.D.: Modeling the spread of rubella disease using the concept of with local derivative with fractional parameter: beta-derivative. Complexity 21(6), 442-451 (2016)

26. Rodrigues, H.S., Monteiro, M.T., Torres, D.F.M.: Dynamics of dengue epidemics when using optimal control. Math. Comput. Model. 52, 1667-1673 (2010)

27. Imran, M., et al.: Mathematical analysis of the role of hospitalization/isolation in controlling the spread of Zika fever. Virus Res. 255, 95-104 (2018)

28. Momoh, A.A., Fuegenschuh, A.: Optimal control of intervention strategies and cost effectiveness analysis for a Zika virus model. Oper. Res. Health Care 18, 99-111 (2018)

29. Agusto, F.B., Khan, M.A.: Optimal control strategies for dengue transmission in Pakistan. Math. Biosci. 305, 102-121 (2018)

30. Fleming, W.H., Richel, R.W.: Deterministic and Stochastic Optimal Control, vol. 1. Springer, New York (1975)

31. Lukes, D.L.: Differential Equations Electronics Resource: Classical to Controlled. Academic Press, New York (1982)

32. Lenhart, S., Workman, J.T.: Optimal Control Applied to Biological Models. CRC Press, Boca Roton (2007)

33. Hamdan, N.I., Kilicman, A.: Analysis of fractional order dengue transmission model: a case study in Malaysia. Adv. Differ. Equ. 2019, 31 (2019)

Submit your manuscript to a SpringerOpen ${ }^{\circ}$ journal and benefit from:

- Convenient online submission

- Rigorous peer review

- Open access: articles freely available online

- High visibility within the field

- Retaining the copyright to your article

Submit your next manuscript at $\boldsymbol{\triangleright}$ springeropen.com 